\title{
The Field Copy Problem: to what Extent do Curvature (Gauge Field) and its Covariant Derivatives Determine Connection (Gauge Potential)?
}

\author{
Mark A. Mostow \\ Department of Mathematics, North Carolina State University, Raleigh, N.C. 27650, USA
}

\begin{abstract}
We show that a connection of a principal bundle is determined up to (global) gauge equivalence by the curvature and its covariant derivatives provided that the infinitesimal holonomy group is of constant dimension and the base space is simply connected. If the dimension of the infinitesimal holonomy group varies, there may be obstructions of a topological nature to the existence of a global or even local gauge equivalence between two connections whose curvatures and covariant derivatives of curvature agree everywhere. These obstructions are analyzed and illustrated by examples.
\end{abstract}

\section{Introduction and Notation}

We follow the definitions and conventions of Kobayashi and Nomizu [7]. Let $P$ be a fixed principal bundle with gauge group $G$ over a base space $M$, and let $\pi: P \rightarrow M$ be the bundle projection. The group $G$ may be any Lie group (we denote its Lie algebra by $\mathfrak{g}$ ), while $M$ and $P$ are smooth $\left(=C^{\infty}\right)$ manifolds, and $\pi$ is a smooth map. We denote the (right) action of $G$ on $P$ by $(u, g) \mapsto u \cdot g \in P$, where $u \in P$ and $g \in G$. Let $\omega$ be a ( $g$-valued) connection 1 -form on $P$ and $\Omega$ its curvature 2 -form, as defined in [7]. In physical terms, $\omega$ is a gauge potential (usually denoted $A$ in physics), and $\Omega$ (denoted $F$ in physics) is the gauge field it determines. If $X$ is any smooth vector field on $M$, let $\tilde{X}$ denote the $(\omega$-)horizontal lift of $X$ to $P$. [Thus $\omega(\tilde{X})=0$ and $\pi_{*} \tilde{X}_{u}=X_{\pi(u)}$ for all $u \in P$.] If $f$ is a $C^{\infty}$ vector-valued function on $P$, one defines the covariant derivative of $f$ along $X$ to equal the usual derivative $\tilde{X} f$ of $f$ along $\tilde{X}$.

Given local coordinates $\left(x_{1}, \ldots, x_{n}\right)$ on an open set $V \subset M$, we let $\partial_{i}=\partial / \partial x_{i}$ and denote $\tilde{\partial}_{i}$ by $D_{i}$, so that $D_{i} f$ is the covariant derivative of $f$ in the $i$ th direction. Now choose any $C^{\infty}$ local trivialization $P \mid V \stackrel{\approx}{\longrightarrow} V \times G$. This is equivalent to choosing a local gauge (section) on $V$, namely, the section corresponding to $V \times\{1\} \subset V \times G$, where 1 is the identity element of $G$. Then the restriction $\omega \mid V \times G$ equals $\theta+\sum_{i=1}^{n} A_{i} d x_{i}$, where $\theta$ is the canonical left-invariant $\mathrm{g}$-valued 1 -form on $G$ and the $A_{i}: V \times G \rightarrow g$ are smooth functions of type $a d$, that is, $A_{i}(u \cdot g)$ 
$=\operatorname{ad}_{g-1}\left(A_{i}(u)\right)$ for all $u \in P \mid V \approx V \times G$ and all $g \in G$. Recall that as a tangent vector on $V \times G, D_{i}=\tilde{\partial}_{i}=\partial_{i}-A_{i}$, but that when we compute the covariant derivative of any function $f: P \rightarrow \mathfrak{g}$ of type ad, $D_{i}$ acts as $\partial_{i}+\operatorname{ad}_{A_{t}(u)}$, that is [7, p. 97]

$$
\left.D_{i} f\right|_{u}=\left.\tilde{\partial}_{i} f\right|_{u}=\left.\partial_{i} f\right|_{u}+\left[A_{i}(u), f(u)\right] \text {. }
$$

The curvature has components

$$
F_{j k}=2 \Omega\left(\tilde{\partial}_{j}, \tilde{\partial}_{k}\right)=\partial_{j} A_{k}-\partial_{k} A_{j}+\left[A_{j}, A_{k}\right],
$$

which are also functions on $V \times G$ of type ad. By covariant derivatives of curvature we mean $D_{i} F_{j k}, D_{i_{1}} D_{i_{2}} F_{j k}$, etc., which are all functions of type ad on $V \times G$ $\left[7\right.$, p. 97]. To be concise, we will let $I$ be a multi-index $\left(i_{1}, \ldots, i_{r}\right)$ of $r$ integers $i_{j}$ between 1 and $n=\operatorname{dim} M$ (here $r=0,1,2, \ldots$ is called the order of $I$ ), and let $D_{I} F_{j k}$ denote $D_{i_{1}} D_{i_{2}} \ldots D_{i_{r}} F_{j k}$. If $I$ is empty, then $D_{I} F_{j k}$ means $F_{j k}$. If $\omega^{\prime}$ is another connection, we let $D_{i}^{\prime}, D_{I}^{\prime}$ denote covariant derivatives with respect to $\omega^{\prime}$, and let $\Omega^{\prime}$ denote the curvature of $\omega^{\prime}$.

A gauge transformation of $P$ is a map $B: P \rightarrow P$ mapping each fiber to itself and satisfying $B(u \cdot g)=B(u) \cdot g$ for all $u \in P, g \in G$. In a local trivialization $V \times G \approx P \mid V$, the map $B$ can be written in the form $B(x, g)=(x, b(x) g)$ for some function $b: V \rightarrow G$. Equivalently, $B=\left(\mathrm{id}, L_{b(x)}\right)$, where for $h \in G, L_{h}: G \rightarrow G$ denotes left multiplication by $h$. Applying $B$ corresponds locally to changing from the gauge (section) $x \mapsto(x, 1)(x \in V)$ to the gauge $x^{\mapsto} \rightarrow(x, b(x))$. We could allow $b$ and $B$ to be $C^{1}$ maps, but as we shall see in Sect. 2 , we might as well assume they are $C^{\infty}$, so we shall do this.

In gauge field theory there has been considerable work to find out how many different potentials $\omega$ can give rise to the same field $\Omega$. If $G$ is abelian, then $\Omega=d \omega$ so that $\Omega^{\prime}=\Omega$ if and only if $\omega^{\prime}-\omega$ is a closed 1 -form. In the case of a non-abelian group $G$, Roskies [9] and Calvo [2] found sufficient conditions on $\Omega$ for $\Omega$ to determine $\omega$ uniquely.

Now two gauge potentials $\omega$ and $\omega^{\prime}$ are considered physically equivalent if they are gauge equivalent (denoted here by $\omega \sim \omega^{\prime}$ ), that is, if there is a gauge transformation $B: P \rightarrow P$ which pulls back the 1-form $\omega^{\prime}$ to $\omega$ [we write then $\left.B^{*}\left(\omega^{\prime}\right)=\omega\right]$, or equivalently, which maps $\omega$-horizontal vectors in $P$ to $\omega^{\prime}$-horizontal vectors. (We say for short that $B$ takes $\omega$ to $\omega^{\prime}$.) Hence another natural question to ask is:

If $\omega$ and $\omega^{\prime}$ have the same curvature $\Omega$, do $\omega$ and $\omega^{\prime}$ have to be gauge equivalent?

In the abelian case the answer is yes if $M$ is simply connected. The reason is that $\omega-\omega^{\prime}$ is then a closed 1-form which can be written in the form $\pi^{*}(d f)$ for some $f: M \rightarrow \mathfrak{g}$; if we define a gauge transformation $B: P \rightarrow P$ by $B(u)$ $=u \cdot \exp (f(\pi(u)))[$ or $B(x, g)=(x, g+\exp (f(x)))$ in coordinates - here $\exp : \mathfrak{g} \rightarrow G$ is the exponential map] - then $B^{*}\left(\omega^{\prime}\right)=\omega^{\prime}+\pi^{*}(d f)=\omega$. In the nonabelian case the answer is no; a counterexample was exhibited in [10]. Thus if $G$ is non-abelian, the curvature alone is insufficient to determine the connection up to gauge equivalence.

If we consider the curvature together with its covariant derivatives we are led to the field copy problem: 
If $\Omega=\Omega^{\prime}$ and all corresponding covariant derivatives of curvature are equal (i.e. in each coordinate chart, $D_{I} F_{j k}=D_{I}^{\prime} F_{j k}^{\prime}$ for all $j, k$ and all multi-indices $I$ of all orders, at all $u \in P$ ), do $\omega$ and $\omega^{\prime}$ have to be gauge equivalent?

$\mathrm{Gu}$ and Yang [6] proved that the answer is no by exhibiting two connections $\omega, \omega^{\prime}$, satisfying the hypotheses, which are not gauge equivalent on $M$. In their example, $\omega$ and $\omega^{\prime}$ are locally gauge equivalent in the sense that $M$ can be covered by open sets $U_{a}$ for which $\omega\left|U_{a} \sim \omega^{\prime}\right| U_{a}$. That is, one can define gauge transformations $B_{a}$ on $P \mid U_{a}=\pi^{-1}\left(U_{a}\right)$ taking $\omega$ to $\omega^{\prime}$, but the $B_{a}$ do not piece together to a global gauge transformation $B$. They believed that this example demonstrated the general behavior, that is, that if $\left(\Omega=\Omega^{\prime}\right.$ and) $D_{I} \Omega=D_{I}^{\prime} \Omega^{\prime}$ for some $\omega$ and $\omega^{\prime}$, then $\omega$ and $\omega^{\prime}$ must be locally gauge equivalent [and may or may not be (globally) gauge equivalent].

It turns out, though, that $\omega$ and $\omega^{\prime}$ are guaranteed to be locally gauge equivalent when $D_{I} \Omega=D_{I}^{\prime} \Omega^{\prime}$ only if several hypotheses are added. (I shall exhibit an example in which $\omega$ and $\omega^{\prime}$ fail to be locally gauge equivalent.) There are topological obstructions both to finding local gauge equivalences and to piecing together local gauge equivalences to a global gauge equivalence. These obstructions depend not on the base space per se but rather on the topology of its subsets on which the dimension of the infinitesimal holonomy group is constant. In this paper I shall solve the field copy problem by analyzing the obstructions and obtaining conditions for $\omega$ and $\omega^{\prime}$ to be locally or globally gauge equivalent.

\section{Results and Examples}

A key role in our results is played by the infinitesimal holonomy group $\phi^{\prime}(u)$ and its Lie algebra $\mathfrak{g}^{\prime}(u)$ (as defined in [7]). Given a connection $\omega$ on $P$ and a point $u \in P, \mathfrak{g}^{\prime}(u)$ is defined to be the linear subspace of $g$ generated by the values of all ( $F_{j k}$ and) $D_{I} F_{j k}$ evaluated at $u$ (this is independent of the choice of coordinates). The facts we shall need about infinitesimal, local, restricted, and global holonomy are listed at the beginning of Sect. 3 below.

We start with a simple well-known result.

Theorem 1. Let $P$ be a $C^{\infty}$ principal bundle with group $G$ over a connected manifold $M$, and let $\omega, \omega^{\prime}$ be two connections on $P$. Suppose $B: P \rightarrow P$ is a $C^{\infty}$ gauge transformation with $B^{*}\left(\omega^{\prime}\right)=\omega$. Then $B$ is uniquely determined by any one of its values $B(u)$, for any fixed $u \in P$.

Proof. Since $B$ takes $\omega$-horizontal vectors to $\omega^{\prime}$-horizontal vectors, it maps $\omega$-horizontal curves to $\omega^{\prime}$-horizontal curves. Thus the value of $B(u)$ determines $B(v)$ uniquely for every point $v$ on every $\omega$-horizontal curve starting at $u$. The fact that $B(v \cdot g)=B(v) \cdot g$ for all $v \in P$ and $g \in G$ determines $B$ of any point of $P$ not already considered. Q.E.D.

Let $B$ be a gauge transformation taking $\omega$ to $\omega^{\prime}$. If $B$ locally equals (id, $L_{b(x)}$ ) (see Sect. 1), then $b$ satisfies the usual partial differential equations

$$
b^{-1} \partial_{i} b=\left(A_{i}-\operatorname{ad}_{b(x)^{-1}} A_{i}^{\prime}\right) \in \mathfrak{g}
$$

where $A_{i}=A_{i}(x, 1)$ and $A_{i}^{\prime}=A_{i}^{\prime}(x, 1)$. (In particular, observe that if $\omega$ and $\omega^{\prime}$ are $C^{\infty}$ and $B$ is $C^{1}$, then $B$ is in fact $C^{\infty}$, since if $b$ is $C^{n}$ then so is $\partial_{i} b$ for all $i$, so that $b$ is $C^{n+1}$.) 
We would like to find necessary and sufficient conditions on $\omega$ and $\omega^{\prime}$ for these partial differential equations to admit a solution $b$, that is, for $\omega$ and $\omega^{\prime}$ to be gauge equivalent (on $V \times G$ ). For necessary conditions, observe that if $\omega=B^{*}\left(\omega^{\prime}\right)$, then

$$
D_{I} F_{j k} \quad \text { at } \quad(x, 1)=\operatorname{ad}_{b(x)^{-1}}\left(D_{I}^{\prime} F_{j k}^{\prime}\right) \text { at }(x, 1) \text { for all } x, I, j, k
$$

[This can be proved easily from the definitions by exploiting the relationships between the pullback $\left(B^{*}\right)$ of the differential forms $\omega^{\prime}$ and $\Omega^{\prime}$ and the pushforward $\left(B_{*}\right)$ of the tangent vector $\tilde{\partial}_{i}$ (see [7]) and by using the fact that $D_{I} F_{j k}$ is of type ad.]

Conversely, suppose we are given $\omega, \omega^{\prime}$, and $b$ for which condition (2) above holds. We want to know if $\omega$ is gauge equivalent to $\omega^{\prime}$. Let $\omega^{\prime \prime}=B^{*} \omega^{\prime}$, where $B$ is the gauge transformation corresponding to $b$. Clearly it is equivalent to find out if $\omega$ and $\omega^{\prime \prime}$ are gauge equivalent. But

$$
D_{I} F_{j k}=D_{I}^{\prime \prime} F_{j k}^{\prime \prime}
$$

Hence our necessary conditions (2) will be sufficient if and only if condition (3) is sufficient. It turns out that condition (3) is sufficient to guarantee a solution only if certain topological conditions are satisfied. To isolate the differential geometry from the topology, we first study the special case in which $\operatorname{dim}^{\prime}(u)$ is constant. We remark that $\mathrm{Gu}$ and Yang [6] have a somewhat different proof of a local version of the following theorem. They do not explicitly assume $\operatorname{dim}^{\prime}(u)$ to be constant, but their proof depends on this assumption or one similar to it.

Theorem 2. Let $P$ be $C^{\infty}$ principal bundle with group $G$ over a simply connected base manifold $M$, and let $\omega$ be a $C^{\infty}$ connection on $P$ for which $\operatorname{dim}^{\prime}(u)$ is constant on $P$. Let $\omega^{\prime}$ be any other $C^{\infty}$ connection on $P$, and suppose that $\omega$ and $\omega^{\prime}$ have identical curvatures and covariant derivatives of curvature, that is, that in each chart, ( $F_{j k}=F_{j k}^{\prime}$ and) $D_{I} F_{j k}=D_{I}^{\prime} F_{j k}^{\prime}$ at all $u \in P$ for all $j, k$, and multi-indices $I$. Then $\omega$ and $\omega^{\prime}$ are (globally) gauge equivalent. In fact, given any $u \in P$ and any $g \in C_{G}\left(\mathfrak{g}^{\prime}(u)\right)$, the centralizer of $\mathfrak{g}^{\prime}(u)$ in $G\left[=\left\{g \in G \mid \operatorname{ad}_{g}(X)=X\right.\right.$ for all $\left.\left.X \in \mathfrak{g}^{\prime}(u)\right\}\right]$, there exists a unique gauge transformation $B: P \rightarrow P$ satisfying (a) $B^{*}\left(\omega^{\prime}\right)=\omega$ and (b) $B(u)=u \cdot g$. Conversely, if $B$ is a gauge transformation satisfying $B^{*}\left(\omega^{\prime}\right)=\omega$ and if $u \in P$, then $B(u)=u \cdot g$ for some $g \in C_{G}\left(\mathfrak{g}^{\prime}(u)\right)$.

Remark. The hypotheses imply that $\omega$ and $\omega^{\prime}$ have the same infinitesimal holonomy Lie algebra $\mathfrak{g}^{\prime}(u)$ at each $u$.

The proof of Theorem 2 will be given in Sect. 3. Now we shall give a generalization of the theorem and discuss some consequences. We shall let $h(\omega, \gamma, u)$ denote the holonomy of a connection $\omega$ around a loop $\gamma$ in $M$ using a reference point $u$ in the fiber of $P$ over $\gamma(0)$ (see Sect. 3 below or [7] for the definition of holonomy).

Theorem 2'. Same hypotheses as Theorem 2, except that $M$ need only be connected. Let $u \in P$ and $x=\pi(u)$. Then there is a right action of the fundamental group $\pi_{1}(M, x)$ on $C_{G}\left(g^{\prime}(u)\right)$, where the latter is regarded only as a manifold [i.e. the action need not respect the group structure of $\left.C_{G}\left(g^{\prime}(u)\right)\right]$. The action is

$$
\left.g[\gamma]=h\left(\omega^{\prime}, \gamma, u\right)\right) g h(\omega, \gamma, u)^{-1},
$$


where $g \in C_{G}\left(\mathfrak{g}^{\prime}(u)\right)$ and $\gamma$ is any piecewise $C^{1}$ loop in the class $[\gamma] \in \pi_{1}(M, x)$. There is a gauge transformation $B: P \rightarrow P$ with $B^{*}\left(\omega^{\prime}\right)=\omega$ and $B(u)=u \cdot g$ if and only if $g$ belongs to $C_{G}\left(g^{\prime}(u)\right)$ and is invariant under the action of $\pi_{1}(M, x)$. (There may not be any such g.)

Remark 1 . Theorem $2^{\prime}$ is related to the general result that any two connections $\omega, \omega^{\prime}$ admit a global gauge equivalence $B$ taking $\omega$ to $\omega^{\prime}$ and $u$ to $u \cdot g$ if and only if $g h(\omega, \gamma, u)=h\left(\omega^{\prime}, \gamma, u\right) g$ for all loops $\gamma$ at $x=\pi(u)$. (This is precisely the condition that allows one to construct $B$ by following the procedure in the proof of Theorem 1.) In the general case one must verify this equality for every loop $\gamma$, since the condition may hold for one loop and fail to hold for a loop homotopic to it. If the hypotheses of Theorem $2^{\prime}$ hold, however, one need only verify (4) for one loop $\gamma$ in each homotopy class.

Remark 2. Although Theorems 2 and $2^{\prime}$ as stated require all the covariant derivatives to be equal, results of Gu and Yang [6] and of Deser and Drechsler [3] show that it suffices to require only that all corresponding covariant derivatives of order $\leqq r$ be equal, where $r$ is some integer depending only on the group $G$.

Remark 3. The right side of formula (4) makes sense for all $g$ in $G$, but it need not be independent of the choice of $\gamma$ within its homotopy class unless $g \in C_{G}\left(g^{\prime}(u)\right)$.

Corollary 1. Let $P$ be a $C^{\infty}$ principal bundle over any manifold $M$, and let $\omega, \omega^{\prime}$ be $C^{\infty}$ connections satisfying $\left(\Omega=\Omega^{\prime}\right.$ and) $D_{I} F_{j k}=D_{I}^{\prime} F_{j k}^{\prime}$ for all $I, j, k$. Suppose that $\mathfrak{g}^{\prime}(u)=\mathfrak{g}$ for all $u \in P$, and that $C_{G}(\mathfrak{g})$, the centralizer of $\mathfrak{g}$, equals $\{1\}$. Then $\omega=\omega^{\prime}$.

Proof. Cover $M$ with open balls $U_{a}$, and let $P_{a}=P \mid U_{a}$. By the theorem, there exists $B_{a}: P_{a} \rightarrow P_{a}$ with $B_{a}^{*}\left(\omega^{\prime} \mid P_{a}\right)=\omega \mid P_{a}$. For any $u \in P_{a}, B_{a}(u)=u \cdot g$ for some $g \in C_{G}\left(g^{\prime}(u)\right)$. But $C_{G}\left(\mathfrak{g}^{\prime}(u)\right)=C_{G}(\mathfrak{g})=\{1\}$, so $g=1$ and $B_{a}(u)=u$. Hence $\omega\left|P_{a}=\mathrm{id}^{*}\left(\omega^{\prime} \mid P_{a}\right)=\omega^{\prime}\right| P_{a}$. Therefore $\omega=\omega^{\prime}$. Q.E.D.

Remark. A slightly stronger statement than Corollary 1 can be proven directly. Replace the hypothesis that the centralizer $C_{G}(\mathfrak{g})$ of $\mathfrak{g}$ in $G$ equal $\{1\}$ by the weaker condition that $z(\mathfrak{g})$, the center of $\mathfrak{g}(=\{X \in \mathfrak{g} \mid[X, Y]=0$ for all $Y \in \mathfrak{g}\})$ equal $\{0\}$. Since $D_{i} f=\partial_{i} f+\left[A_{i}, f\right]$ for each $\mathfrak{g}$-valued function of type ad on $U \times G \subset P$ $[7$, p. 97] we have

$$
0=D_{i}^{\prime} D_{I}^{\prime} F_{j k}^{\prime}-D_{i} D_{I} F_{j k}=\left(D_{i}^{\prime}-D_{i}\right) D_{I} F_{j k}=\left[A_{i}^{\prime}-A_{i}, D_{I} F_{j k}\right]
$$

Hence $A_{i}^{\prime}-A_{i}$ commutes with $\operatorname{span}\left\{D_{I} F_{j k}\right\}=\mathfrak{g}$. Therefore $A_{i}^{\prime}-A_{i} \in z(\mathfrak{g})=\{0\}$. Hence $A_{i}^{\prime}=A_{i}$ and $\omega=\omega^{\prime}$. Q.E.D.

Corollary 2. Let $P$ be an analytic principal bundle over a simply connected manifold $M$, and let $\omega, \omega^{\prime}$ be $C^{\infty}$ connections on $P$ for which ( $\Omega=\Omega^{\prime}$ and) $D_{I} F_{j k}=D_{I}^{\prime} F_{j k}^{\prime}$. If $\omega$ is analytic ( $\omega^{\prime}$ need not be), then $\omega$ and $\omega^{\prime}$ are gauge equivalent.

Proof. In this case, $\operatorname{dim}^{\prime}(u)$ is constant [7], so that Theorem 2 applies. Q.E.D.

Remark. Gu and Yang [6] proved a result (their Theorem 4) similar to Corollary 2, using a different method.

Let us now apply Theorems 2 and $2^{\prime}$ to the field copy problem in the general case, in which $\operatorname{dim} g^{\prime}(u)$ need not be constant on $P$. 
Thus suppose that $\omega, \omega^{\prime}$ are any $C^{\infty}$ connections on $P$ (with arbitrary connected base manifold $M$ ) and that $D_{I} F_{j k}=D_{I}^{\prime} F_{j k}^{\prime}$ for all $I, j, k$ (in every chart). Let $\left\{V_{a}\right\}$ be the collection of maximal connected open subsets of $M$ on which $\operatorname{dimg}^{\prime}$ is constant. Pick a point $u_{a}$ in each $P_{a}=\pi^{-1}\left(V_{a}\right)$ and let $x_{a}=\pi\left(u_{a}\right)$. Let $S_{a}=C_{G}\left(g^{\prime}\left(u_{a}\right)\right)$ if $V_{a}$ is simply connected; if $\pi_{1}\left(V_{a}\right) \neq\{1\}$, let $S_{a}$ be the set of elements of $C_{G}\left(\mathfrak{g}\left(u_{a}\right)\right)$ which are invariant under the action of $\pi_{1}\left(V_{a}, x_{a}\right)\left(S_{a}\right.$ may be empty). By Theorems 2 and $2^{\prime}$, the elements of $S_{a}$ are in one to one correspondence with the gauge transformations taking $\omega \mid P_{a}$ to $\omega^{\prime} \mid P_{a}$. For $g \in S_{a}$, let $B_{a, g}$ denote the unique gauge transformation of $P_{a}$ for which $B_{a, g}^{*}\left(\omega^{\prime} \mid P_{a}\right)=\omega \mid P_{a}$ and $B_{a, g}\left(u_{a}\right)=u_{a} \cdot g$. The problem of finding a global gauge equivalence $B$ between $\omega$ and $\omega^{\prime}$ reduces to finding choices of $g_{a} \in S_{a}$ for each $a$ in such a way that the map $\cup_{a} B_{a, g_{a}}$ mapping $\cup_{a} P_{a}$ to itself can be extended to a $C^{1}$ (and hence $C^{\infty}$ ) map $B: P \rightarrow P$. Such an extension $B$ is unique if it exists, since $\cup_{a} P_{a}$ is dense in $P$, as one can show easily using the lower semicontinuity of $\operatorname{dimg}^{\prime}$ (or see [8, p. 108]). Moreover, $B$ is determined by its value at any one point of $P$, by Theorem 1 .

We illustrate these ideas now with examples. For concreteness let $P=R^{2} \times \mathrm{SO}(3)$. Identify $\mathfrak{g}=\mathfrak{s o}(3)$ with the three-dimensional cross-product Lie algebra and let $\left\{e_{1}, e_{2}, e_{3}\right\}$ correspond to the standard basis of that algebra. For example, we may let

$$
e_{1}=\left(\begin{array}{rrr}
0 & 0 & 0 \\
0 & 0 & -1 \\
0 & 1 & 0
\end{array}\right) \quad e_{2}=\left(\begin{array}{rrr}
0 & 0 & 1 \\
0 & 0 & 0 \\
-1 & 0 & 0
\end{array}\right) \quad e_{3}=\left(\begin{array}{rrr}
0 & -1 & 0 \\
1 & 0 & 0 \\
0 & 0 & 0
\end{array}\right)
$$

(see [1, p. 24]). Let $\left(x_{1}, x_{2}\right)$ be the standard coordinates on $R^{2}$. Let $f: R \rightarrow R$ be any $C^{\infty}$ function for which $f^{-1}(0)$ is some closed interval $q \leqq x \leqq r$. Let $h: R \rightarrow R$ be any $C^{\infty}$ function with $h(x) \neq 0$ if and only if $q<x<r$. Define a connection $\omega^{\prime}=\theta+\sum A_{i}^{\prime} d x_{i}$ by

$$
\begin{aligned}
A_{1}^{\prime}\left(\left(x_{1}, x_{2}\right), g\right) & =\operatorname{ad}_{g^{-1}}\left(f\left(x_{1}\right)\left(e_{1}+x_{2} e_{2}\right)\right) \\
A_{2}^{\prime} & =0 .
\end{aligned}
$$

Define another connection $\omega$ by

$$
\begin{aligned}
A_{1}\left(\left(x_{1}, x_{2}\right), g\right) & =A_{1}^{\prime}\left(\left(x_{1}, x_{2}\right), g\right)+\operatorname{ad}_{g^{-1}}\left(h\left(x_{1}\right) e_{1}\right) \\
A_{2} & =0 .
\end{aligned}
$$

The curvature $\Omega^{\prime}$ has one component

$$
\begin{aligned}
F_{12}^{\prime} & =F^{\prime}=\partial_{1} A_{2}^{\prime}-\partial_{2} A_{1}^{\prime}+\left[A_{1}^{\prime}, A_{2}^{\prime}\right] \\
& =-f\left(x_{1}\right) e_{2} \quad\left[\text { at }\left(\left(x_{1}, x_{2}\right), 1\right)\right]
\end{aligned}
$$

while

$$
F=F^{\prime} \text {. }
$$


Now, acting on functions of type ad, the covariant derivative operators at $\left(\left(x_{1}, x_{2}\right), 1\right)$ are

$$
\begin{aligned}
& D_{1}^{\prime}=\partial_{1}+\operatorname{ad}_{A_{1}^{\prime}} \\
& D_{2}^{\prime}=\partial_{2}=D_{2} \\
& D_{1}=D_{1}^{\prime}+\operatorname{ad}_{h\left(x_{1}\right) e_{1}} .
\end{aligned}
$$

Define open sets $V_{1}, V_{2}, V_{3}$ in $R^{2}$ by the conditions $x_{1}<q$, respectively $q<x_{1}<r$, respectively $x_{1}>r$. On $V_{1} \cup V_{3}, h\left(x_{1}\right)=0$, so that $D_{1}=D_{1}^{\prime}$. On $V_{2}, f\left(x_{1}\right)=0$, so that $F^{\prime}=F=0$. It follows that $D_{I} F=D_{I}^{\prime} F^{\prime}$ for all multi-indices $I$ on $V_{1} \cup V_{2} \cup V_{3}$, and hence on $R^{2}$, by continuity.

The infinitesimal holonomy Lie algebra $\mathfrak{g}^{\prime}\left(\left(x_{1}, x_{2}\right), 1\right)$ equals 0 on $V_{2}$ and $\mathfrak{g}$ on $V_{1} \cup V_{3}$. Hence its centralizer $G_{x_{1}, x_{2}}=C_{G}\left(g\left(\left(x_{1}, x_{2}\right), 1\right)\right)$ equals $G$ on $V_{2}$ and $\{1\}$ on $V_{1} \cup V_{3}$. By the previous discussion, the gauge transformations of $V_{2} \times G$ taking $\omega$ to $\omega^{\prime}$ are in one-to-one correspondence with the elements of $G=\mathrm{SO}(3)$, while on $V_{1} \times G$ or $V_{3} \times G$ only the identity map takes $\omega$ to $\omega^{\prime}$. Suppose there is a global gauge transformation $B$ on $P$ taking $\omega$ to $\omega^{\prime}$. Such a map must be the identity on $\left(V_{1} \cup V_{3}\right) \times G$. If $B=\left(\mathrm{id}, L_{b\left(x_{1}, x_{2}\right)}\right)$, then $b: R^{2} \rightarrow G$ must satisfy the partial differential equations

$$
\begin{aligned}
& \partial_{1} b=A_{1}-\mathrm{ad}_{b\left(x_{1}, x_{2}\right)^{-1}} \mathrm{~A}_{1}^{\prime} \quad\left[\text { at }\left(\left(x_{1}, x_{2}\right), 1\right)\right] \\
& \partial_{2} b=0 .
\end{aligned}
$$

Hence $b=b\left(x_{1}\right)$, and $b\left(x_{1}\right)=1 \in G$ for $x_{1} \leqq q$ or $x_{1} \geqq r$ (using continuity for $x_{1}=q$ or $r$ ).

For $q<x_{1}<r$ we get

$$
\partial_{1} b=h\left(x_{1}\right) e_{1} \quad\left(\text { at }\left(\left(x_{1}, x_{2}\right), 1\right)\right] .
$$

The unique solution satisfying $b(q)=1 \in G$ is

$$
b\left(x_{1}\right)=\exp \left(\left(\int_{q}^{x_{1}} h(t) d t\right) e_{1}\right) \in G .
$$

Letting $H\left(x_{1}\right)=\int_{q}^{x_{1}} h(t) d t$, we compute

$$
b\left(x_{1}\right)=\left(\begin{array}{ccc}
1 & 0 & 0 \\
0 & \cos \left(H\left(x_{1}\right)\right) & -\sin \left(H\left(x_{1}\right)\right) \\
0 & \sin \left(H\left(x_{1}\right)\right) & \cos \left(H\left(x_{1}\right)\right)
\end{array}\right) .
$$

Thus $b(r)=1$ is satisfied if and only if $H(r)=2 \pi n$ for some integer $n$. Since the latter is not usually true, we see that in general, no global gauge transformation $B$ taking $\omega$ to $\omega^{\prime}$ can exist. Our argument shows, however, that $B$ can always be defined on $\left\{x_{1}<r\right\}$ or on $\left\{x_{1}>q\right\}$. Also, our constructed $b(x)$ always equals 1 for $x_{1} \leqq q$, and if $b(r)=1$, then $b\left(x_{1}\right)=1$ for all $x_{1} \geqq r$. Hence $\omega \sim \omega^{\prime}$ on $R^{2}$ if and only if $H(r)=2 \pi n$.

This example can be modified to construct $\omega$ and $\omega^{\prime}$, again on $R^{2} \times \mathrm{SO}(3)$, with $D_{I} F_{j k}=D_{I}^{\prime} F_{j k}^{\prime}$, which are not gauge equivalent even when restricted to an arbitrarily small neighborhood of $(0,0)$ in $R^{2}$. Indeed, the formulas for $A_{i}$ and $A_{i}^{\prime}$ 
remain the same, but we change the functions $f$ and $h$. For $n=1,2, \ldots$ let $V_{n}=\{x \in R \mid 1 /(n+1) \leqq x \leqq 1 / n\}$. Let $V_{0}=\{1 \leqq x\}$, and $V_{-2}=\{x \leqq 0\}$. Choose $f$ to be any $C^{\infty}$ function from $R$ to itself which is zero precisely on those $V_{n}$ with $n$ odd and at the point 0 . Let $h$ be a non-negative $C^{\infty}$ function which is zero precisely on those $V_{n}$ with $n$ even. These choices are possible since for any closed set $C \subset R^{n}$ there exists a non-negative $C^{\infty}$ function $k: R^{n} \rightarrow R$ with $k^{-1}(0)=C[4$, p. 17]. Now suppose there existed a neighborhood $U$ of $(0,0)$ and a gauge transformation $B$ taking $\omega \mid U \times G$ to $\omega^{\prime} \mid U \times G$. For any large enough even $n$ and some open interval $J$ around $0, U$ contains $\left(V_{n} \cup V_{n+1} \cup V_{n+2}\right) \times J$. But by the previous example, $B$ can be defined on this set if and only if $\int_{V_{n+1}} h(t) d t$ is a multiple of $2 \pi$. Since these integrals are all positive $[(n+1)$ is odd $]$ and approach 0 as $n \rightarrow \infty$, it follows that for large enough $n$ the integrals are never multiples of $2 \pi$. Hence $B$ cannot be defined on $U$.

The foregoing examples illustrate that the configuration of the open sets $V_{a} \subset M$ on which dimg $g^{\prime}$ is constant strongly influences the existence of a gauge transformation $B$ taking $\omega$ to $\omega^{\prime}$ when $D_{I} F_{j k}=D_{I}^{\prime} F_{j k}^{\prime}$. We shall now find conditions on $\left\{V_{a}\right\}$ which guarantee that such a $B$ always exists globally, respectively locally. We shall content ourselves with convenient sufficient conditions only, since the enormous variety of configurations possible seems to preclude any set of simple necessary and sufficient conditions. (See Remark 1 after Theorem 3.)

Definition. Let $M$ be a $C^{\infty}$ manifold and let $\left\{V_{a}\right\}$ be a collection of disjoint, open, connected subsets of $M$ whose union is dense in $M$. The collection $\left\{V_{a}\right\}$ is said to be good if

1. There are only finitely many $V_{a}$.

2. Each $V_{a}$ is simply connected.

3. Each $\bar{V}_{a}$ is a $C^{1}$ submanifold of $M$ with corners, having interior $V_{a}$ and boundary $\bar{V}_{a}-V_{a}$. That is, on some neighborhood $U$ of any point of $\bar{V}_{a}-V_{a}$ there is a $C^{1}$ chart of $M$ under which the set

$$
\left\{\left(x_{1}, x_{2}, \ldots, x_{n}\right) \in R^{n} \mid x_{j}>0, j=1,2, \ldots, k\right\}
$$

for some $1<k \leqq n$ (which we shall call a sector) corresponds to $V_{a} \cap U$, while its closure corresponds to $\bar{V}_{a} \cap U$.

4. The $\bar{V}_{a}$ have at least one common intersection point $x$.

5. For all $V_{a}, V_{b}$, every point $y \in \bar{V}_{a} \cap \bar{V}_{b}$ is connected to $x$ by a piecewise $C^{1}$ path lying entirely in $\bar{V}_{a} \cap \bar{V}_{b}$. In particular, $\bar{V}_{a} \cap \bar{V}_{b}$ is connected.

Definition. Let $M,\left\{V_{a}\right\}$ be as before. Then $\left\{V_{a}\right\}$ is said to be locally good if $M$ can be covered by open sets $U$ each having the property that the collection of all components of $V_{a} \cap U$ is good in $U$.

Theorem 3. Let $P$ be a $C^{\infty}$ principal bundle with group $G$ over any base manifold $M$. Let $\omega, \omega^{\prime}$ be connections on $P$ for which $D_{I} F_{j k}=D_{I}^{\prime} F_{j k}^{\prime}$ in each chart and at all $u \in P$, for all $I, j, k$. Let $\left\{V_{a}\right\}$ be the components of the interiors of the subsets of $M$ on which $\operatorname{dim} g^{\prime}=0$, respectively $1,2, \ldots$, dimg. (More generally, let $\left\{V_{a}\right\}$ be a collection of disjoint, connected, open subsets of $M$ whose union is dense in $M$ and on each of which dimg' is constant.) Then

a) If $\left\{V_{a}\right\}$ is good then $\omega$ and $\omega^{\prime}$ are always (i.e. with no more conditions on $\omega$ and $\omega^{\prime}$ ) gauge equivalent (globally on $P$ ).

b) If $\left\{V_{a}\right\}$ is locally good then $\omega$ and $\omega^{\prime}$ are always locally gauge equivalent on $P$. 
Remarks. 1. The converses are false. For example, if we separate $R^{2}$ into four regions by removing the $x_{1}$-axis and the parabola $x_{2}=x_{1}^{2}$, we get a configuration which is not good, since no $C^{1}$ chart in a neighborhood of the origin can map either "wedge" onto a quadrant of $R^{2}$, as required. Nonetheless, the conclusions of Theorem 3 are true for this configuration.

2. In the example given in which there was no global gauge equivalence, $\left\{V_{i}\right\}$ failed to be good because $\left\{\bar{V}_{i}\right\}$ lacks a common point of intersection. In the example of no local gauge equivalence, the basic problem was the lack of local finiteness of $\left\{V_{a}\right\}$ near $\left\{x_{1}=0\right\}$. This caused there to be no common point of intersection. Away from $\left\{x_{1}=0\right\}$, the collection is locally good.

\section{Proofs}

We will need the following facts about holonomy. Their proofs are collected in [7].

1. $\mathfrak{g}^{\prime}(u)$ is a sub-Lie algebra of $\mathfrak{g}$.

2. $\mathfrak{g}^{\prime}(u \cdot g)=\operatorname{ad}_{g^{-1}}\left(\mathfrak{g}^{\prime}(u)\right)$ (hence $\operatorname{dim} \mathfrak{g}^{\prime}$ is constant on each fiber of $P$ ).

3. If $u \in P$ and $\gamma$ is a piecewise $C^{1}$ path in $M$ starting at $x=\pi(u)$, let $\tilde{\gamma}$ denote the $(\omega$-)horizontal lift of $\gamma$ to a path in $P$ starting at $\tilde{\gamma}(0)=u$. If $\gamma$ is a loop in $M$, then $\tilde{\gamma}(1)$ $=u \cdot g$ for some $g \in G$, and $h(\omega, \gamma, u)($ def. $)=g$ is called the holonomy of $\omega$ around $\gamma$ with reference point $u$. For fixed $u$ and $\omega,\{h(\omega, \gamma, u)\} \subset G$ is called the holonomy group $\phi(u)$ of $\omega$ with reference point $u$, while the subgroup $\phi^{0}(u)=\{h(\omega, \gamma, u) \mid \gamma$ is contractible $\}$ is the restricted holonomy group. For any open neighborhood $V$ of $x$ in $M$, let $\phi^{0}(u, V)=\{h(\omega, \gamma, u) \mid \gamma$ is a loop contractible in $V\}$. Then $\phi^{*}(u)$, the local holonomy group of $\omega$ at $u$, is defined to be the intersection $\cap \phi^{0}(u, V)$ over all connected open neighborhoods $V$ of $x$. The Lie algebras of $\phi^{*}(u), \phi^{0}(u), \phi(u)$ are denoted $\mathfrak{g}^{*}(u), \mathfrak{g}(u), \mathfrak{g}(u)$, respectively (the latter two are equal).

4. $\mathfrak{g}^{\prime}(u) \subset \mathfrak{g}^{*}(u) \subset \mathfrak{g}(u)$.

5. The dimension $\operatorname{dim}^{\prime}(u)$, regarded as an integer-valued function on $M$, is lower semi-continuous. That is, for each integer $m,\left\{\pi(u) \in M \mid \operatorname{dim}^{\prime}(u) \geqq m\right\}$ is open.

6. If $\operatorname{dim}^{\prime}(u)$ is constant on $P$, then $\mathfrak{g}^{\prime}(u)=\mathfrak{g}^{*}(u)=\mathfrak{g}(u)$ for all $u \in P$, and $\phi^{\prime}(u)$ $=\phi^{*}(u)=\phi^{0}(u)$.

7. If $P$ and $\omega$ are real analytic and $M$ is connected, then $\operatorname{dim}^{\prime}(u)$ is constant.

8. If $u, v \in P$ are connected by an $\omega$-horizontal path in $P$, then $\mathfrak{g}(u)=\mathfrak{g}(v)$. It follows that if $U$ is an open set in $M$ with $\operatorname{dimg}^{\prime}$ constant on $P \mid U$, and if $u, v$ are connected by a horizontal path in $P \mid U$, then $\mathfrak{g}^{\prime}(u)=\mathfrak{g}^{\prime}(v)$.

Proof of Theorem 2. First we prove the theorem locally, using a local trivialization $P \mid U \approx R^{n} \times G$. Without loss of generality, we may take our reference point $u_{0}$ to be $(0,1) \in R^{n} \times G$. By the discussion after Theorem 1 , a $C^{\infty}$ gauge transformation on $P \mid U$ with $B^{*}\left(\omega^{\prime}\right)=\omega$ and $B\left(u_{0}\right)=u_{0} \cdot g_{0}$ exists if and only if there exists a $C^{1}$ function $b: R^{n} \rightarrow G$ satisfying the system of partial differential equations

$$
b(x)^{-1} \partial_{i} b=A_{i}(x, 1)-\operatorname{ad}_{b(x)^{-1}} A_{i}^{\prime}(x, 1) \in \mathfrak{g}=T G_{1}
$$

with initial condition $b(0)=g_{0}$ [for then we can define $\left.B(x, g)=(x, b(x) g)\right]$. To simplify the computations we shall think of $G$ as a matrix group and $g$ as a matrix Lie algebra, but it is possible to complete the proof for any $G$ without doing so. Thus we can write

$$
\partial_{i} b=b(x) A_{i}(x)-A_{i}^{\prime}(x) b(x) \in T G_{b(x)},
$$


where $A_{i}(x)$ means $A_{i}(x, 1)$, etc. and $b A_{i}$ and $A_{i}^{\prime} b$ are computed by matrix multiplication. Now if a solution $b(x)$ existed, then its graph $\Gamma=\{(x, b(x))\}$ would be a submanifold of $R^{n} \times G$. (Remark: This $R^{n} \times G$ should not be identified with $P \mid U$. It is, rather, a local trivialization of $\operatorname{Ad} P \mid U$, where $\operatorname{Ad} P$ is the bundle over $M$ with fiber $G$ associated [7, p. 55] to $P$ by the adjoint action of $G$ on $G$. Ad $P$ is not a principal bundle.) The tangent space of $\Gamma$ at $(x, b(x))$ would be spanned by vectors

$$
X_{i}(x, b(x))=\partial_{i}+\partial_{i} b=\partial_{i}+b(x) A_{i}(x)-A_{i}^{\prime}(x) b(x) \in T\left(R^{n} \times G\right)_{(x, b(x))} .
$$

Let us therefore define vector fields $X_{i}$ on all of $R^{n} \times G$ by

$$
X_{i}(x, g)=\partial_{i}+g A_{i}(x)-A_{i}^{\prime}(x) g .
$$

Let $\mathscr{X}(x, g)$ be the linear subspace of $T\left(R^{n} \times G\right)_{(x, g)}$ spanned by $\left\{X_{i}(x, g)\right\}_{i=1}^{n}$. Then $\mathscr{X}$ is a $C^{\infty} n$-dimensional distribution (=field of $n$-planes) on $R^{n} \times G$. A graph $\Gamma$ as above containing $\left(0, g_{0}\right) \in R^{n} \times G$ exists if and only if $\mathscr{X}$ admits an integral submanifold through $\left(0, g_{0}\right)$ which projects down to $R^{n}$ (under $\pi$ ) in a one-to-one and onto manner.

We check first if $\mathscr{X}$ is integrable. The Theorem of Frobenius [7, p. 10] says that $\mathscr{X}$ is completely integrable (admits integral submanifolds through every point of $\left.R^{n} \times G\right)$ if and only if $\left[\left[X_{j}, X_{k}\right]\right]_{(x, g)}$ always lies in $\mathscr{X}(x, g)$. Here we write $[[]$,$] to$ denote the bracket product of vector fields, as distinguished from $[$,$] , the Lie$ bracket on $g$. But $\pi_{*}\left(X_{j}(x, g)\right)=\partial_{j} \in T R_{x}^{n}$, so that $\pi_{*}\left(\left[\left[X_{j}, X_{k}\right]\right]\right)=\left[\left[\partial_{j}, \partial_{k}\right]\right]=0$. Hence $\left[\left[X_{j}, X_{k}\right]\right]_{(x, g)}$ is a vertical vector. But the only linear combination $\sum c_{i} X_{i}(x, g)$ which is vertical is the zero vector. Therefore $\mathscr{X}$ is completely integrable if and only if $\left[\left[X_{j}, X_{k}\right]\right]=0$ for all $j, k$. Breaking up this bracket product into terms, we compute that $\left[\left[g A_{j}(x), g A_{k}(x)\right]\right]=g\left[A_{j}(x), A_{k}(x)\right]$, since on each fiber $\{x\} \times G$, $g A_{j}$ and $g A_{k}$ are left-invariant vector fields. On the other hand, [ $\left.\left[A_{j}^{\prime} g, A_{k}^{\prime} g\right]\right]$ $=-\left[A_{j}^{\prime}(x), A_{k}^{\prime}(x)\right] g$. (Proof: The flow generated by $A_{j}^{\prime} g$ is $(t, g) \mapsto \exp \left(t A_{j}^{\prime}\right) g$. By $[7$, p. 15],

$$
\begin{aligned}
{\left[\left[A_{j}^{\prime} g, A_{k}^{\prime} g\right]\right] } & =\lim _{t \rightarrow 0} t^{-1}\left(A_{k}^{\prime} g-\exp \left(t A_{j}^{\prime}\right) A_{k}^{\prime} \exp \left(-t A_{j}^{\prime}\right) g\right) \\
& =-\left(\frac{d}{d t} \operatorname{ad}_{\exp \left(t A_{j}^{\prime}\right)}\left(A_{k}^{\prime}\right)\right) g \\
& =-\left(\operatorname{ad}_{A_{j}^{\prime}}\left(A_{k}^{\prime}\right)\right) g \\
& \left.=-\left[A_{j}^{\prime}, A_{k}^{\prime}\right] g .\right)
\end{aligned}
$$

Furthermore, $\left[\left[g A_{j}(x), A_{k}^{\prime}(x) g\right]\right]=0$, since the flow of $g A_{j}(x)$, respectively of $A_{k}^{\prime}(x) g$, on $\{x\} \times G$ is a one-parameter family of right, respectively left, translations of $G$, so that the two flows commute (see [7, p. 16]). Also, $\left[\left[\partial_{j}, g A_{k}(x)\right]\right]=\partial_{j}\left(g A_{k}(x)\right)$ $=g \partial_{j} A_{k}$. Collecting terms, we get

$$
\begin{aligned}
{\left[\left[X_{j}, X_{k}\right]\right] } & =g\left(\partial_{j} A_{k}-\partial_{k} A_{j}+\left[A_{j}, A_{k}\right]\right)-\left(\partial_{j} A_{k}^{\prime}-\partial_{k} A_{j}^{\prime}+\left[A_{j}^{\prime}, A_{k}^{\prime}\right]\right) g \\
& =g F_{j k}-F_{j k}^{\prime} g \in T\left(R^{n} \times G\right)_{(x, g)} .
\end{aligned}
$$

Hence the condition $\left[\left[X_{j}, X_{k}\right]\right]=0$ is satisfied only at those $(x, g)$ for which $g F_{j k}(x)$ $=F_{j k}^{\prime}(x) g$, that is, where

$$
F_{j k}(x, 1)=\operatorname{ad}_{g^{-1}} F_{j k}^{\prime}(x, 1)
$$


Let $Q_{0}$ denote the subset of $R^{n} \times G$ satisfying this condition. Now $\mathscr{X}$ satisfies the Frobenius condition on $Q_{0}$, but $\mathscr{X}$ need not be tangent to $Q_{0}$. Indeed, if $X_{i}$ is tangent to some curve in $Q_{0}$ at $(x, g)$, then $\left[\left[X_{i}, g F_{j k}-F_{j k}^{\prime} g\right]\right]=0$ at $(x, g)$. We compute, in a manner similar to that of the previous calculation,

$$
\begin{aligned}
0 & =g \partial_{i} F_{j k}(x)-\partial_{i} F_{j k}^{\prime}(x) g+\left[\left[g A_{i}(x)-A_{i}^{\prime}(x) g, g F_{j k}(x)-F_{j k}^{\prime}(x) g\right]\right] \\
& =g \partial_{i} F_{j k}-\partial_{i} F_{j k}^{\prime} g+g\left[A_{i}(x), F_{j k}(x)\right]-\left[A_{i}^{\prime}, F_{j k}^{\prime}\right] g \\
& =g D_{i} F_{j k}-D_{i}^{\prime} F_{j k}^{\prime} g .
\end{aligned}
$$

Thus all the $X_{i}$ are tangent to $Q_{0}$ at $(x, g)$ only if

$$
(x, g) \in Q_{1}=(\text { def. })\left\{(x, g) \in Q_{0} \mid D_{i} F_{j k}(x)=\operatorname{ad}_{g^{-1}} D_{i}^{\prime} F_{j k}^{\prime}(x) \text { for all } i, j, k\right\} .
$$

Define

$$
\begin{gathered}
Q_{r}=\left\{(x, g) \in R^{n} \times G \mid D_{I} F_{j k}(x)=\operatorname{ad}_{g^{-1}} D_{I}^{\prime} F_{j k}^{\prime}(x)\right. \\
\text { for all } j, k, \text { and all } I \text { of order } \leqq r\} .
\end{gathered}
$$

A similar calculation shows that the $X_{i}$ are tangent to curves in $Q_{r}$ at $(x, g)$ only if $(x, g) \in Q_{r+1}$. Finally, let $Q=\cap_{r} Q_{r}$. Since $D_{I} F_{j k}=D_{I}^{\prime} F_{j k}^{\prime}$ by hypothesis, and the linear span of the $D_{I} F_{j k}(x)$ is $\mathrm{g}^{\prime}(x, 1)$, we see that

$$
Q=\left\{(x, g) \in R^{n} \times G \mid g \in C_{G}\left(\mathfrak{g}^{\prime}(x, 1)\right)\right\} .
$$

We must answer a crucial question before can apply Frobenius' Theorem to $\mathscr{X} \mid Q$ :

Is $Q$ a submanifold of $R^{n} \times G$ ?

Since $\operatorname{dim} \mathfrak{g}^{\prime}()$ is constant by hypothesis, $\mathfrak{g}^{\prime}(u)=\mathfrak{g}^{\prime}(v)$ if $u, v \in P$ are connected by a horizontal path (by property 8 of $\mathfrak{g}^{\prime}$ ). For each $x \in R^{n}$, let $\gamma^{x}$ be the (radial) path $t^{\mapsto} \rightarrow t x$ from 0 to $x$, and let $t \mapsto\left(t x, f^{x}(t)\right) \in R^{n} \times G$ be its $\omega$-horizontal lift starting at $(0,1) \in R^{n} \times G$. One can show that $f^{x}(1)$ depends smoothly on $x$. We have $g^{\prime}(0,1)$ $=\mathfrak{g}^{\prime}\left(x, f^{x}(1)\right)=\operatorname{ad}_{f^{x}(1)^{-1}}\left(\mathfrak{g}^{\prime}(x, 1)\right)$ (by property 2 of $\left.\mathfrak{g}^{\prime}\right)$. Thus $\mathfrak{g}^{\prime}(x, 1)=\operatorname{ad}_{f^{x}(1)}\left(\mathfrak{g}^{\prime}(0,1)\right)$, and $C_{G}\left(g^{\prime}(x, 1)\right)=\operatorname{ad}_{f^{x}(1)}\left(C_{G}\left(\mathfrak{g}^{\prime}(0,1)\right)\right)$. The map from $R^{n} \times G$ to itself defined by $(x, g) \mapsto\left(x, \operatorname{ad}_{f^{x(1)}} g\right)$ is a $C^{\infty}$ diffeomorphism mapping $R^{n} \times C_{G}\left(g^{\prime}(0,1)\right)$ onto $Q$. Therefore $Q$ is a $C^{\infty}$ submanifold of $R^{n} \times G$.

Now we can conclude by our earlier calculations that $\mathscr{X}$ is tangent to $Q$ and that $\mathscr{X} \mid Q$ satisfies the Frobenius condition. Therefore, through every point of $Q$ there passes a unique maximal $C^{\infty}$ submanifold (called a leaf of $\mathscr{X}$ ) of dimension $n$ which is tangent to $\mathscr{X}$. Since $\pi_{*}$ maps $\mathscr{X}_{(x, g)}$ isomorphically onto $T R_{x}^{n}$, the restriction of $\pi: R^{n} \times G \rightarrow R^{n}$ to any leaf $L \subset Q$ is a local diffeomorphism (at every point of $L$ ), by the Inverse Function Theorem. I claim that in fact $\pi$ maps $L$ diffeomorphically onto $R^{n}$.

To see this, we shall use a sort of "analytic continuation" of gauge transformations (the word analytic is used only to evoke a similar construction in complex analysis: we continue to assume only $C^{\infty}$ smoothness rather than actual analyticity of maps). Let $\gamma$ be any $C^{1}$ path in $R^{n}$. Let $(\gamma(0), g)$ be a point in $Q$, and let $L$ be the leaf containing $(\gamma(0), g)$. By the preceding results, there is an open neighborhood $V$ of $\gamma(0)$ and a map $b: V \rightarrow G$ such that the map $x \mapsto(x, b(x))$ is a local smooth inverse to $\pi \mid L$. By the remarks at the beginning of the proof, $b$ gives rise to a gauge transformation $B_{V}$ taking $\omega \mid V$ to $\omega^{\prime} \mid V$. On the other hand, the construction in the proof of Theorem 1 yields a function $\beta:[0,1] \rightarrow G$ with the 
property that if a gauge transformation $B$ exists which takes $\omega$ to $\omega^{\prime}$ and maps $(\gamma(0), 1)$ to $(\gamma(0), g)$, then $B(\gamma(t), 1)=(\gamma(t), \beta(t))$ for all $t \in[0,1]$. Pick a number $\tau>0$ so that for all non-negative $t<\tau, \gamma(t) \in V$. The uniqueness of $B$ implies that $\beta(t)=b(\gamma(t))$ for all $t \in[0, \tau]$. For all $t \in[0, \tau]$ the point $(\gamma(t), \beta(t))$ lies in the leaf $L$, so by continuity, $(\gamma(\tau), \beta(\tau))$ also lies in $L$. We have shown that the set $\{t \mid(\gamma(t), \beta(t)) \in L\}$ is open, closed, and nonempty. Hence $(\gamma(t), \beta(t))$ lies in $L$ for all $t \in[0,1]$. Thus we see that the map $\pi \mid L: L \rightarrow R^{n}$ has the property of unique path lifting (as do covering spaces [5]). By lifting radial paths in $R^{n}$ up to $L$ we can construct an explicit inverse $s: R^{n} \rightarrow L$ to the map $\pi \mid L$. Hence $L \subset R^{n} \times G$ is the desired graph $\Gamma$, and the desired gauge equivalence is the function $b: R^{n} \rightarrow G$ satisfying $s(x)=(x, b(x))$. This completes the proof of the Theorem in the case that the base space $M$ is $R^{n}$.

The proof of the Theorem for arbitrary simply connected base manifolds $M$ is similar, with the following modifications. The submanifold $Q$ is contained not in $R^{n} \times G$ or in $M \times G$ but rather in the bundle $\operatorname{Ad} P$ (over $M$ ) alluded to earlier in the proof. We can think of a point $w \in \operatorname{Ad} P$ in the fiber over $x \in M$ as being an automorphism $w: P_{x} \rightarrow P_{x}$ of the fiber of $P$ over $x$. That is, $w(u \cdot g)=w(u) \cdot g \in P_{x}$ for all $u \in P_{x}, g \in G$. If $R^{n} \times G$ is a local trivialization of $P \mid V$, then $w$ acts as a left multiplication, i.e. there exists some $h \in G$ (depending on $w$ and the trivialization) for which $w(x, g)=(x, h g)$ for all $g \in G$. Clearly a gauge transformation of $P$ is precisely a global section of $\operatorname{Ad} P$. The map $\pi \mid L: L \rightarrow M$ is shown to be a covering space map (see [5] for the definition of and basic results about covering spaces). Since $M$ is simply connected by hypothesis, $\pi \mid L$ is a homeomorphism, by covering space theory. Its inverse map $s: M \rightarrow L \subset \operatorname{Ad} P$ is the desired global gauge transformation. Q.E.D. Theorem 2.

Proof of Theorem 2'. The proof of Theorem 2 carries over almost completely. We find in the end that $\operatorname{Ad} \pi \mid L: L \rightarrow M$ is a covering map, but not necessarily a bijection. Given any $C^{1}$ path $\gamma$ in $M$ starting at $x_{0}$ and given a point $w_{0} \in L$ lying over $x_{0}$, there is a unique lift $\gamma_{w_{0}}$ of $\gamma$ to $\operatorname{Ad} P$ lying in $L$ and satisfying $\gamma_{w_{0}}(0)=w_{0}$. For $L \rightarrow M$ to be a bijection, it is necessary and sufficient that $\gamma_{w_{0}}(1)=\gamma_{w_{0}}(0)=w_{0}$ for all $C^{1}$ loops $\gamma$ at $x_{0}$. By the theory of covering spaces, $\gamma_{w_{0}}(1)$ is independent of the choice of $\gamma$ within its homotopy class (leaving endpoints fixed).

Pick $u_{0} \in$ (fiber of $P$ over $x_{0}$ ). Let $g_{0} \in G$ be the group element for which $w_{0}\left(u_{0}\right)$ $=u_{0} \cdot g_{0}$. As before, $g_{0} \in C_{G}\left(\mathfrak{g}^{\prime}\left(u_{0}\right)\right)$. Given $\gamma$, let $\tilde{\gamma}_{1}$, respectively $\tilde{\gamma}_{2}$, be the $\omega$-, respectively $\omega^{\prime}$-horizontal lifts of $\gamma$ starting at $u_{0}$, respectively $u_{0} \cdot g_{0}$. Then

1. By definition of holonomy around a loop, $\tilde{\gamma}_{1}(1)=u_{0} \cdot h\left(\omega, \gamma, u_{0}\right)$, while

$$
\tilde{\gamma}_{2}(1)=\left(u_{0} \cdot g_{0}\right) \cdot h\left(\omega^{\prime}, \gamma, u_{0} g_{0}\right)=u_{0} g_{0} \operatorname{ad}_{g_{0}{ }_{1}} h\left(\omega^{\prime}, \gamma, u_{0}\right)
$$

$[7$, p. 72$]$

$$
=u_{0} h\left(\omega^{\prime}, \gamma, u_{0}\right) g_{0}
$$

2. By the construction of $\gamma_{w_{0}}$, we have $\tilde{\gamma}_{2}(t)=\gamma_{w_{0}}(t)\left(\tilde{\gamma}_{1}(t)\right)$ for all $t$.

3. Pick a local trivialization for $P$ over a neighborhood of $x_{0}$ in which $u_{0}$ corresponds to $(0,1) \in R^{n} \times G$. Then $\tilde{\gamma}_{1}(1)=\left(0, h\left(\omega, \gamma, u_{0}\right)\right), \tilde{\gamma}_{2}(1)=\left(0, h\left(\omega^{\prime}, \gamma, u_{0}\right) g_{0}\right)$, and $\gamma_{w_{0}}(1)$ is left multiplication of $G$ by

$$
H_{\gamma}\left(g_{0}\right)=(\text { def. }) h\left(\omega^{\prime}, \gamma, u_{0}\right) g_{0} h\left(\omega, \gamma, u_{0}\right)^{-1} .
$$


The maps $H_{\gamma}: G \rightarrow G$ have the following properties:

a) $H_{\gamma}$ maps $C_{G}\left(\mathfrak{g}^{\prime}\left(u_{0}\right)\right)$ to itself.

Proof. Since $\tilde{\gamma}_{1}(1)$ and $\tilde{\gamma}_{2}(1) \in\left\{(0, g) \mid g \in C_{G}\left(\mathfrak{g}^{\prime}\left(u_{0}\right)\right)\right\}$, and since $C_{G}\left(\mathfrak{g}^{\prime}\left(u_{0}\right)\right)$ is a group, we must have $H_{\gamma}\left(g_{0}\right) \in C_{G}\left(\mathfrak{g}^{\prime}\left(u_{0}\right)\right)$.

b) If $\gamma, \delta$ are piecewise $C_{1}$ loops in $M$ at $x_{0}$ and $\gamma * \delta$ is the concatenation $\gamma$, then $\delta$, then

$$
H_{\gamma * \delta}\left(g_{0}\right)=H_{\delta}\left(H_{\gamma}\left(g_{0}\right)\right) \text { for all } g_{0} \in C_{G}\left(\mathfrak{g}^{\prime}\left(u_{0}\right)\right) .
$$

c) If $\gamma$ and $\delta$ are homotopic loops at $x_{0}$, and if $g_{0} \in C_{G}\left(g^{\prime}\left(u_{0}\right)\right)$, then $H_{\gamma}\left(g_{0}\right)=H_{\delta}\left(g_{0}\right)$.

Proof. By construction, $H_{\gamma}\left(g_{0}\right)$ is the endpoint of the lift of $\gamma$ to the covering space $L \rightarrow M$, where $L$ is the leaf of $\mathscr{X}$ containing the $w_{0} \in \operatorname{Ad} P$ satisfying $w_{0}\left(u_{0}\right)=u_{0} \cdot g_{0}$. By covering space theory, $H_{\gamma}\left(g_{0}\right)$ depends only on the homotopy class of $\gamma$. Q.E.D. by

In summary, $\pi_{1}\left(M, x_{0}\right)$ acts on the set $C_{G}\left(\mathfrak{g}^{\prime}\left(u_{0}\right)\right)$ (we ignore its group structure)

$$
g_{0}[\gamma]=H_{\gamma}\left(g_{0}\right) .
$$

The covering map $L \rightarrow M$ is a bijection if and only if $g_{0}$ is invariant under the action, that is, if and only if

$$
h\left(\omega^{\prime}, \gamma, u_{0}\right) g_{0} h\left(\omega, \gamma, u_{0}\right)^{-1}=g_{0}
$$

for all piecewise $C^{1}$ loops $\gamma$ in $M$ at $x_{0}$. It suffices to verify this condition for a collection of generators of $\pi_{1}\left(M, x_{0}\right)$. Q.E.D. Theorem $2^{\prime}$.

Sketch of proof of Theorem 3. Part (b) will follow immediately from part (a), which we shall now prove. Following the discussion after Theorems 2 and $2^{\prime}$ and their corollaries, we let $S_{a}$ denote the set of gauge transformations taking $\omega \mid V_{a}$ to $\omega^{\prime} \mid V_{a}$. We think of these as sections of $\operatorname{Ad} P \mid V_{a}$. Our problem is to choose an $s_{a}$ from each $S_{a}$ in such a way that $\cup_{a} S_{a}$ can be extended to a global $C^{\infty}$ section of $\operatorname{Ad} P$. Using the hypothesis that $\left\{V_{a}\right\}$ is good, and applying techniques similar to those used in the proofs of Theorems 1, 2, and 2', we prove the following:

1. Every $s \in S_{a}$ can be extended uniquely to a section $\bar{s}$ of $\operatorname{Ad} P \mid \bar{V}_{a}$, where $\bar{V}_{a}$ is the closure of $V_{a}$.

2. For any fixed $a$ and fixed $y \in \bar{V}_{a}$, the set of values $S_{a, y}=\left\{\bar{s}(y) \in \operatorname{Ad} P \mid s \in S_{a}\right\}$ is a subgroup of the fiber of $\operatorname{Ad} P$ over $y$.

3. For any $s_{a} \in S_{a}$ and any $s_{b} \in S_{b}$, if $\bar{s}_{a}$ and $\bar{s}_{b}$ agree at one point $x \in \bar{V}_{a} \cap \bar{V}_{b}$, then they agree on all of $\bar{V}_{a} \cap \bar{V}_{b}$.

4. By definition of goodness there is a point $x$ common to every $\bar{V}_{a}$. By Step 3, the possible values of $\bar{s}_{a}(x)$ (a fixed) form a subgroup $S_{a, x}$ of the fiber of $\operatorname{Ad} P$ over $x$. The intersection $S_{x}=\cap_{a} S_{a, x}$ is not empty, since it contains 1 . Pick any $w_{0} \in S_{x}$, and choose an $\bar{s}_{a}$ for each $a$ so that $\bar{s}_{a}(x)=w_{0}$. By Step 3, $\bar{s}_{a}$ and $\bar{s}_{b}$ agree on $\bar{V}_{a} \cap \bar{V}_{b}$. Therefore $\cup_{a} \bar{s}_{a}$ defines a function $s: \cup_{a} \bar{V}_{a}=M \rightarrow \operatorname{Ad} P$. Since each restriction $s \mid \bar{V}_{a}=\bar{S}_{a}$ is continuous, and since $\left\{\bar{V}_{a}\right\}$ is a finite closed cover of $M, s$ is continuous.

5. The map $s$ is $C^{1}$ (and hence $C^{\infty}$, by the discussion after Theorem 1). Q.E.D. Theorem 3. 
Acknowledgements. I wish to acknowledge valuable discussions with Richard Palais. The exposition benefited from comments and questions posed by participants in the Yang-Mills Seminar at North Carolina State University and the Mathematical Physics Seminar at Brandeis University. Thanks are due to Stanley Deser for bringing to my attention the work of $\mathrm{Gu}$ and Yang on this problem, which I was not aware of when I did the research on which this paper is based.

\section{References}

1. Belinfante, J., Kolman, B.: A survey of Lie groups and Lie algebras with applications and computational methods. Philadelphia: SIAM 1972

2. Calvo, M. : Phys. Rev. D 15, 1733-1735 (1977)

3. Deser, S., Drechsler, W.: Generalized gauge field copies (preprint)

4. Golubitsky, M., Guillemin, V.: Stable mappings and their singularities. Berlin, Heidelberg, New York: Springer 1973

5. Greenberg, M.: Lectures on algebraic topology. New York: W. A. Benjamin, Inc. 1967

6. Gu, C.-H., Yang, C.-N.: Sci. Sin. 20, 47-55 (1977)

7. Kobayashi, S., Nomizu, K.: Foundation of differential geometry, Vol. 1. New York: Interscience Publishers 1963

8. Lichnerowicz, A.: Global theory of connections and holonomy groups. Leyden: Noordhoff International Publishing 1976

9. Roskies, R.: Phys. Rev. D 15, 1731-1732 (1977)

10. Wu, T.-T., Yang, C.-N.: Phys. Rev. D 12, 3843 (1975)

Communicated by A. Jaffe

Received January 11, 1980; in revised form June 20, 1980 